\title{
Le traitement chirurgical des adénocarcinomes de la jonction œsogastrique: Expérience marocaine à travers une série de 149 cas
}

\author{
Glaoui Meryem ${ }^{1, \&}$, Naciri Sarah ${ }^{1}$, Ghanem Samia ${ }^{1}$, Belkouchi Abdelkader ${ }^{2}$, Errihani Hassan ${ }^{1}$ \\ ${ }^{1}$ Service d'oncologie medicale, Institut National d'Oncologie, Rabat, Maroc, ${ }^{2}$ Service de chirurgie viscérale A, CHU Ibn Sina, Rabat, Maroc \\ ${ }^{\&}$ Corresponding author: Glaoui Meryem, Institut National d'Oncologie, Hay Irfane, Rabat, Maroc
}

Key words: Cardia, adénocarcinome, Siewert, chirurgie, Maroc

Received: 01/02/2011 - Accepted: 29/03/2011 - Published: 30/03/2011

\begin{abstract}
Introduction: L'incidence du cancer de la Jonction œsogastrique (JOG) ne cesse d'augmenter depuis les deux dernières décades aussi bien dans les pays industrialisés que dans les pays en voie de développement notamment le Maroc. Le rapprochement sur le plan étiopathogénique des adénocarcinomes (ADK) du cardia et ceux du bas œsophage reste un sujet de controverse posant le problème du choix thérapeutique chirurgical, notamment l'étendu de la résection. Le but de ce travail est de dresser le profil épidémiologique des patients opérés pour un ADK du cardia et analyser les gestes chirurgicaux réalisés par l'équipe chirurgicale A du centre hospitalier universitaire IBN SINA à Rabat à travers une série de 149 cas. Méthodes: Il s'agit d'une étude rétrospective ayant intéressé les malades opérés pour un ADK de la JOG sur une période de 15 années (1990-2004) en chirurgie A du CHU IBN SINA à Rabat. Résultats: 149 cas d'ADK de la JOG ont été retenus. L'âge moyen était de 55 ans, $76 \%$ étaient de sexe masculin avec un sex-ratio de $3 / 1$. Les signes cliniques les plus fréquemment observés sont la dysphagie (70\%), les douleurs épigastriques (67\%) et le reflux gastro-oesophagien (15.5\%). La notion de tabagisme n'a été rapportée que chez $20 \%$ des cas et l'œsophage de barret chez $10 \%$ des patients. Le type I de Siewert a concerné 65 cas (43.5\%), le type II 40 cas (27 \%), et le type III 44 cas (29.5\%). Dans le type I une œsophagectomie transhiatale a été proposée, alors que les type II et III ont été traité comme un cancer de l'estomac par une gastrectomie totale. Les suites opératoires étaient simples chez $80 \%$ des patients, la mortalité globale était de $8.5 \%$. Conclusion: L'œsophagectomie par voie transhiatale chez les patients fragiles avec un ADK de la JOG de type I permet des résultats carcinologiques satisfaisants avec réduction de la morbidité postopératoire par rapport à la voie transthoracique. La gastrectomie totale est le traitement de choix pour les types III, alors que le débat est toujours ouvert quant à la meilleure stratégie chirurgicale pour la prise en charge des tumeurs de type II.
\end{abstract}

\section{Pan African Medical Journal. 2011; 8:35}

This article is available online at: http://www.panafrican-med-journal.com/content/article/8/35/full/

(c) Glaoui Meryem et al. The Pan African Medical Journal - ISSN 1937-8688. This is an Open Access article distributed under the terms of the Creative Commons Attribution License (http://creativecommons.org/licenses/by/2.0), which permits unrestricted use, distribution, and reproduction in any medium, provided the original work is properly cited. 
La fréquence des adénocarcinomes de la jonction œsogastrique augmente de façon alarmante, plus rapidement que $n$ 'importe quel autre cancer en Europe [1]. Ces derniers différent des cancers épidérmoides de l'œsophage non seulement par l'histologie mais aussi en raison d'une histoire naturelle et pronostic différents [2,3], Ils différent également des adénocarcinomes gastriques sous-jacents par une épidémiologie propre, un envahissement lymphatique précoce, une propagation bidirectionnelle vers le Médiastin et l'abdomen [4] et un pronostic plus sombre [5].

Cette entité a été démembrée par la classification de Siewert et al [6] en 3 types : type I (ou adénocarcinome du bas œsophage, dont le centre est situé $1 \mathrm{~cm}$ au-dessus de l'épicentre de la tumeur), type II (ou adénocarcinome du cardia anatomique, dont le centre est situé entre $1 \mathrm{~cm}$ audessus et $2 \mathrm{~cm}$ en dessous de l'épicentre de la tumeur), et type III (adénocarcinome du fundus étendu au cardia, dont le centre est situé $2 \mathrm{~cm}$ en dessous de l'épicentre de la tumeur). Cette classification est utile en termes de choix thérapeutique chirurgical puisque le type I est traité comme un cancer de l'œsophage alors que le type III rejoint le traitement du cancer de l'estomac, le traitement des types II est toujours controversé [7].

Le but de ce travail rétrospectif est de rapporter les caractéristiques épidémiologiques des malades opérés pour un ADK de la JOG et d'analyser les différents gestes chirurgicaux effectués en fonction de la classification de Siewert.

\section{Méthodes}

Il s'agit d'une étude rétrospective ayant intéressé les malades opérés pour un adénocarcinome (ADK) de la jonction œsogastrique (JOG) sur une période de 15 années (1990-2004) en chirurgie A du CHU IBN SINA à Rabat. Nous avons inclus les ADK intéressant le cardia anatomique et dont le centre est situé à moins de $5 \mathrm{~cm}$ de celui-ci. L'absence d'un endobrachyœsophage (EBO) ou d'une métaplasie intestinale est facultative puisque I'on considère actuellement que la progression tumorale peut détruire ces lésions. Ont été exclu de cette étude, les carcinomes épidérmoides (CE) et les autres types histologiques, ainsi que les ADK du 1/3 supérieur de l'estomac dont le centre est situé à plus de $5 \mathrm{~cm}$ de la JOG. Au total, nous avons retenu 149 cas.

\section{Résultats}

L'étude a porté sur 113 hommes (76\%) et 36 femmes (24\%), le sex-ratio était de 3/1. L'âge variait de 22 à 84 ans avec un âge médian de 55 ans. Parmi les antécédents des patients inclus dans notre série on note la notion de RGO chez 23 malades (15.5\%), d'EBO chez 16 malades (10.7 $\%$ ) et d'Intoxication alcoolo tabagique chez 39 malades (26\%). Le délai moyen au diagnostic était de 8 mois (1 à 24 mois). Les circonstances de découverte étaient dominés par : la dysphagie (70\%), les épigastralgies (67\%), l'altération de l'état général (48 \%), les hématémèses (31\%) et les vomissements (28\%).

Le diagnostic a été établi chez tous nos patients sur des biopsies par FOGD. Selon la classification de Siewert ; 65 malades étaient de type I (43.5\%), 40 malades de type II (27\%) et 44 malades (29.5\%) de type III. La tumeur était localisée chez 104 patients, localement avancée chez 29 patients et métastatique chez 17 patients.

Tous les patients considérés comme ayant un stade localisé étaient opérés d'emblée, le taux de résecabilité était de $70 \%$. Dans le type I une œsogastréctomie transhiatale a été proposée, alors que les type II et III ont été traité comme un cancer de l'estomac par une gastrectomie totale .Les différentes interventions réalisées, les voies d'abord ainsi que le type de rétablissement de continuité sont représentées respectivement dans les tableaux 1, 2 et 3 . En outre, un geste associé à une exérèse majeure a été réalisé chez 3 malades chez qui une extension locorégionale fut découverte en per-opératoire ; ainsi, une splénectomie isolée a été faite chez 2 malades et une splénopancréatectomie chez 1 malade.

Le curage ganglionnaire le plus souvent réalisé était de type curage D1,5 comportant les ganglions périgastriques du curage D1 et une partie des ganglions du curage D2 (coronaire stomachique, bord gauche de l'artère hépatique commune, artère splénique), ainsi, $23 \%$ de curage de type D1 ont été réalisé et $67 \%$ de curage D1,5. Le taux d'exérèse Ro à l'analyse anatomopathologique des pièces opératoires a été de $62 \%$.

Les suites opératoires ont été simples chez 84 malades réséqués pour ADK du cardia. Une ou plusieurs complications sont survenues chez 20 malades (Tableau 4). Nous avons déploré 8 décès, la mortalité globale a été de $8.5 \%$, les causes du décès sont dominées par les complications d'ordre respiratoires (tableau 5).

La durée moyenne d'hospitalisation des patients dans notre institution était de 27 jours chez l'ensemble des opérés. Les variables associées à l'allongement de la durée d'hospitalisation sont essentiellement celles qui sont liées aux complications anastomotiques et/ou respiratoires.

\section{Discussion}

Les adénocarcinomes du bas œsophage, du cardia et de l'estomac sont souvent regroupés ou confondus en une seule entité pour des raisons de simplification ou par pragmatisme du fait de leur similitude histologique [8]. Ainsi, la prise en charge thérapeutique de l'adénocarcinome de l'œsophage est voisine de celle des cancers épidermoïdes, alors que celle de l'adénocarcinome du cardia est plutôt assimilée à celle des cancers gastriques.

Les résultats des récentes séries chirurgicales comportant essentiellement ou exclusivement des adénocarcinomes du cardia ont montré que leur pronostic est plus sombre [9]. 
L'analyse dans la littérature des différents gestes chirurgicaux réalisés dans les ADK de la JOG prend en considération la classification de Siewert. En effet, pour les types I, Un seul essai randomisé a testé la place d'une voie d'abord transthoracique par comparaison à une voie transhiatale pour la réalisation d'une œsogastréctomie chez 220 patients présentant un adénocarcinome du bas œsophage [10]. Avec un suivi en médian de 4,7 ans, l'analyse des résultats anatomopathologiques a montré que la qualité de l'exérèse était équivalente entre les deux techniques (R0 contre R1 contre R2) [11]. La mortalité post opératoire était similaire dans les deux groupes, alors que les complications respiratoires étaient significativement plus élevées dans le groupe avec une voie d'abord transthoracique (57\% vs 27\%; p12].

Dans notre série, l'œsogastréctomie par voie transhiatale a été l'intervention de choix pour les tumeurs de type I de Siewert, La préférence pour cette intervention revient au fait que la majorité des patients étaient fragiles, dénutris et dont l'état pleuro-pulmonaire n'était pas toujours satisfaisant et l'éviction de la thoracotomie permettrait de réduire la morbidité post opératoire liée aux complications respiratoires.

Pour les ADK de la JOG de type II, le débat est toujours ouvert quant au meilleur traitement chirurgical. Une étude multicentrique française a démontré que le taux d'exérèse R0 après gastrectomie totale pour les ADK de la JOG de type II localement avancés (T3-T4) était inferieur par rapport à une oesogastrectomie polaire supérieure $(64,6 \%$ vs $78,7 \%)$ sans différence significative en terme de morbidité ou de mortalité postopératoire $[13,14]$.

Quant à l'équipe de Siewert, ils ont proposé pour le type II, une gastrectomie totale avec œsogastréctomie partielle par voie transhiatale. Une œsogastréctomie polaire supérieure par voie transhiatale n'a été proposée que si une exérèse de type R0 ne pouvait être réalisée par voie abdominale seule $[6,15]$.

Le traitement chirurgical des ADK du cardia de type III est communément apparenté aux tumeurs gastriques et traitées par une gastrectomie totale étendue associée à une résection transhiatale de l'œsophage distal avec une anastomose œsojéjunaleou œsocolique avec coloplastie [16]. Les tumeurs de type III sont de pronostic défavorable par comparaison aux tumeurs de type I et II, de survie après chirurgie équivalente [17].

En termes de curage ganglionnaire pour les adénocarcinomes de la JOG, trois études randomisées n'ont pas permis de mettre en évidence de supériorité en termes de survie du curage ganglionnaire D2 par comparaison au curage D1 (correspondant aux ganglions paracardiaux, de la petite et de la grande courbure, ganglions supra- et infra pyloriques) [18-20]. Néanmoins, les résultats d'une importante étude prospective allemande ayant inclus 1654 patients opérés pour un cancer gastrique avec un curage D1 (moins de 25 ganglions) ou un curage D2 (plus de 25 ganglions) ont démontré que le curage ganglionnaire D2 améliorait significativement la probabilité de survie globale à dix ans de $26 \%$ pour les patients atteints d'une tumeur de stade II [21].

Au terme de ces résultats : I'association française de chirurgie recommande pour le type I de Siewert une oesogastrectomie polaire supérieure par voie transthoracique avec un curage deux champs : médiastinal et abdominal, la voie transhiatale n'étant réservée qu'aux patients fragiles et en cas de contre-indication à la thoracotomie.

Deux approches chirurgicales peuvent être proposées pour les ADK de la JOG de type II: une œsogastréctomie totale par voie abdominale ou une œsogastréctomie polaire supérieure par voie transthoracique ou transhiatale associées à un curage ganglionnaire médiastinal et abdominal.

La gastrectomie totale étant le traitement de choix pour les tumeurs de type III associé à un curage de type D2 sans splénopancreatectomie étant responsable de la morbidité et de la mortalité du curage D2, celle-ci peut être réservée uniquement à la présence de ganglions macroscopiquement envahis le long de l'artère splénique ou dans le cas d'une tumeur envahissant la grosse tubérosité et la séreuse.

\section{Conclusion}

Le choix de la technique chirurgicale dans les ADK de la JOG est controversé. Les résultats de notre série confirment que l'œsogastréctomie par voie transhiatale peut être une alternative intéressante à la thoracotomie chez les malades fragiles avec des résultats carcinologiques similaires. La discussion est toujours ouverte quant à l'étendue de la résection œsophagienne et gastrique pour les tumeurs de type II. L'oesogastrectomie totale avec anastomose œsojéjunale sur anse en Y par voie abdominale pure, avec ou sans ouverture du hiatus reste le traitement de choix des tumeurs de type III.

\section{Conflits d'intérêts}

Les auteurs ne déclarent aucun conflit d'intérêt.

\section{Contribution des auteurs}

Tous les auteurs ont contribué à l'élaboration de ce travail et ont lu et approuvé la version finale du manuscrit. 
Tableau 1: les différents types d'interventions réalisées lors du traitement chirurgical des adénocarcinomes de la jonction œesogastrique dans une série marocaine.

Tableau 2: les voies d'abord utilisées lors du traitement chirurgical des adénocarcinomes de la jonction œsogastrique dans une série marocaine.

Tableau 3: les types d'anastomoses réalisées lors du traitement chirurgical des adénocarcinomes de la jonction œsogastrique dans une série marocaine.

Tableau 4: causes de mortalité postopératoire du traitement chirurgical des adénocarcinomes de la jonction œsogastrique dans une série marocaine.

Tableau 5: morbidité postopératoire lors du traitement chirurgical des adénocarcinomes de la jonction œsogastrique dans une série marocaine.

\section{Références}

1. Triboulet JP, Fabre S, Castel B, Toursel H. Adenocarcinomas of the distal esophagus and cardia: surgical management. Cancer Radiother. 2001 Nov;5 Suppl 1:90s-97s. This article on PubMed

2. Mariette C, Finzi L, Piessen G, Van Seuningen I, Triboulet JP. Oesophageal carcinoma: prognostic differences between squamous cell carcinoma and adenocarcinoma. World J Surg. 2005 Jan;29(1):39-45. This article on PubMed

3. Rohatgi PR, Swisher SG, Correa AM, Wu TT, Liao Z, Komaki R, Walsh GL, Vaporciyan AA, Rice DC, Bresalier RS, Roth JA, Ajani JA. Histologic subtypes as determinants of outcome in oesophageal carcinoma patients with pathologic complete response after preoperative chemoradiotherapy. Cancer. 2006 Feb 1;106(3):552-8. This article on PubMed

4. Goldblum JR. The significance and etiology of intestinal metaplasia of the oesogastric junction. Ann Diagn Pathol. 2002 Feb;6(1):67-73. This article on PubMed

5. Sant M, Aareleid T, Berrino F, Bielska Lasota M, Carli PM, Faivre J, Grosclaude P, Hédelin G, Matsuda T, Moller H, Moller T, Verdecchia A, Capocaccia R, Gatta G, Micheli A, Santaquilani M, Roazzi P, Lisi D; EUROCARE Working Group. EUROCARE-3: survival of cancer patients diagnosed 1990-94--results and commentary. Ann Oncol. 2003;14 Suppl 5:v61-118. This article on PubMed

6. Rudiger Siewert J, Feith M, Werner M, Stein HJ. Adenocarcinoma of the esophagogastric junction: results of surgical therapy based on anatomical/topographic classification in 1002 consecutive patients. Ann Surg. 2000 Sep;232(3):353-61. This article on PubMed

7. Christophe Mariette, Guillaume Piessen, Nicolas Briez. Oesophagogastric junction adenocarcinoma: which therapeutic approach?. Lancet Oncol. 2011 Mar;12(3):296-305. This article on PubMed

8. Buc E, Carrere C, Pezet D. Cancer de la jonction oesogastrique-cancer de l'estomac: la même pathologie?. Hépato-Gastro. 2007 JuilletAout; 14(4)

9. Sant M, Aareleid T, Berrino F, Bielska Lasota M, Carli PM, Faivre J, Grosclaude P, Hédelin G, Matsuda T, Moller H, Moller T, Verdecchia A, Capocaccia R, Gatta G, Micheli A, Santaquilani M, Roazzi P, Lisi D; EUROCARE Working Group. EUROCARE-3: survival of cancer patients diagnosed 1990-94--results and commentary. Ann Oncol. 2003;14 Suppl 5:v61-118. This article on PubMed

10. Hulscher JB, van Sandick JW, de Boer AG, Wijnhoven BP, Tijssen JG, Fockens P, Stalmeier PF, ten Kate FJ, van Dekken H, Obertop H, Tilanus HW, van Lanschot JJ. Extended transthoracic resection compared with limited transhiatal resection for adenocarcinoma of the oesophagus. N Engl J Med. 2002 Nov 21;347(21):1662-9. This article on PubMed

11. Créhange $G$, Bonnetain $F$, Chauffert $B$, Rat $P$, Bedenne $L$, Maingon $P$. Resectable adenocarcinoma of the oesophagogastric junction care: which perioperative treatment?. Cancer Radiother. 2008 Sep;12(5):365-73. This article on PubMed

12. Omloo JM, Lagarde SM, Hulscher JB, et al. Extended transthoracic resection compared with limited transhiatal resection for adenocarcinoma of the mid/distal esophagus: five-year survival. Ann Surg. 2007; 246: 992-1000. This article on PubMed

13. Sauvanet A, Mariette C, Triboulet JP. Cancer du cardia. Rapport l'AFC 2003. Ed Arnette

14. Sauvanet A, Mariette C, Thomas P, Lozac'h P, Segol P, Tiret E, Delpero JR, Collet D, Leborgne J, Pradère B, Bourgeon A, Triboulet JP. Mortality and morbidity after resection for adenocarcinoma of the gastroesophageal junction: predictive factors. J Am Coll Surg. 2005; 201: 253-62. This article on PubMed

15. Feith M, Stein HJ, Siewert JR. Adenocarcinoma of the esophagogastric junction: surgical therapy based on 1602 consecutive resected patients. Surg Oncol Clin N Am. 2006; 15: 751-64. This article on PubMed 
16. Marsman WA, Tytgat GN, ten Kate FJ, van Lanschot JJ. Differences and similarities of adenocarcinomas of the oesophagus and oesophagogastric junction. J Surg Oncol. 2005;92:160-8. This article on PubMed

17. Wijnhoven BP, Siersema PD, Hop WC, van Dekken H, Tilanus HW, Rotterdam Oesophageal Tumour Study Group. Adenocarcinomas of the distal oesophagus and gastric cardia are one clinical entity. Br J Surg. 1999;86:529-35. This article on PubMed

18. Bonenkamp JJ, Hermans J, Sasako M, van de Velde CJ, Welvaart K, Songun I, Meyer S, Plukker JT, Van Elk P, Obertop H, Gouma DJ, van Lanschot JJ, Taat CW, de Graaf PW, von Meyenfeldt MF, Tilanus H; Dutch Gastric Cancer Group. Extended lymph-node dissection for gastric cancer. N Engl J Med. 1999 Mar 25;340(12):908-14. This article on PubMed

19. Cuschieri A, Weeden S, Fielding J, Bancewicz J, Craven J, Joypaul V, Sydes M, Fayers P. Patient survival after D1 and D2 resections for gastric cancer: long-term results of the MRC randomized surgical trial - Surgical Co-operative Group. Br J Cancer. 1999 Mar;79(910):1522-30. This article on PubMed

20. Hartgrink $\mathrm{HH}$, van de Velde $\mathrm{CJ}$, Putter $\mathrm{H}$, Bonenkamp JJ, Klein Kranenbarg E, Songun I, Welvaart K, van Krieken JH, Meijer S, Plukker JT, van Elk PJ, Obertop H, Gouma DJ, van Lanschot JJ, Taat CW, de Graaf PW, von Meyenfeldt MF, Tilanus H, Sasako M. Extended lymph node dissection for gastric cancer: who may benefit? Final results of the randomized Dutch gastric cancer group trial. J Clin Oncol. 2004 Jun 1;22(11):2069-77. This article on PubMed

21. Siewert JR, Bottcher K, Stein HJ, Roder JD. Relevant prognostic factors in gastric cancer: 10-year results of the German gastric cancer Study. Ann Surg. 1998;228:449-61. This article on PubMed

Tableau 1: les différents types d'interventions réalisées lors du traitement chirurgical des adénocarcinomes de la jonction œsogastrique dans une série marocaine

\begin{tabular}{lcc}
\hline Gestes réalisés & Nombre & \% \\
\hline Oesophagectomie transhiatale (OTH) & 60 & 58 \\
Gastrectomie totale (GT) & 30 & 28.5 \\
Oesogastrectomie polaire superieure (OGPS) & 11 & 10.5 \\
Lewis santy & 2 & 2 \\
Akiyama & 1 & 1 \\
Total & 104 & 100 \\
\hline
\end{tabular}

Lewis-Santy : oeso-gastrectomie +anastomose oesogastrique intrathoracique, Akiyama : Oeso-gastrectomie+ Anastomose oesogastrique au cou.

Tableau 2 : Les voies d'abord utilisées lors du traitement chirurgical des adénocarcinomes de la jonction œsogastrique dans une série marocaine

\begin{tabular}{lcc}
\hline Voie d'abord & Nombre & $\%$ \\
\hline Abdomen seul & 41 & $39 \%$ \\
Abdomen+cou & 60 & $58 \%$ \\
Abdomen + thorax Droite & 2 & $2 \%$ \\
Abdomen+Thorax+cou & 1 & $1 \%$ \\
\hline
\end{tabular}


Tableau 3: les types d'anastomoses réalisées lors du traitement chirurgical des adénocarcinomes de la jonction œsogastrique dans une série marocaine

\begin{tabular}{lcc}
\hline Type d'anastomose & Nombre & \% \\
\hline Oeso-colique, cou & 44 & 42.5 \\
Oeso-gastrique, cou & 17 & 16.5 \\
Oeso-jéjunale / anse Y & 19 & 18 \\
Oeso-jéjunale / anse ' $\Omega$ & 2 & 2 \\
Oeso-jéjunale / Henley & 9 & 8.5 \\
Oeso-gastrique, thorax & 2 & 2 \\
Oeso-gastrique, abd & 11 & 10.5 \\
\hline
\end{tabular}

Tableau 4 : causes de mortalité postopératoire du traitement chirurgical des adénocarcinomes de la jonction œsogastrique dans une série marocaine

\begin{tabular}{lccccc}
\hline Causes & OTH & GT & OGPS & LS & Akiyama \\
\hline Péritonite postopératoire & - & 2 & - & - & - \\
Respiratoire & 1 & 1 & 1 & - & - \\
Hémorragie & 1 & - & - & - & - \\
Occlusion & - & 1 & - \\
\hline
\end{tabular}

OTH : Oesophagectomie transhiatale, GT : Gastrectomie totale, OGPS : Oesogastrectomie polaire superieure, LS : Lewis Santy

Tableau 5: morbidité postopératoire lors du traitement chirurgical des adénocarcinomes de la jonction œsogastrique dans une série marocaine

\begin{tabular}{lccccc}
\hline Causes & OTH & LS & Akiyama & GT & OGPS \\
\hline Péritonite postopératoire & 2 & - & - & 2 & - \\
Paralysie recurentielle & 2 & - & - & - & - \\
Fistule cervicale & 3 & - & - & 2 & - \\
Fistule digestive & - & - & - & - & - \\
Abcès sous phrénique & 1 & - & - & 1 & - \\
Médiastinite & - & 1 & - & - \\
Occlusion & - & - & - & - \\
Hémorragie & 1 & - & - & 2 \\
Pleurésie & 1 & - & 1 & 2 \\
Pneumopathie & 2 & 1 & -
\end{tabular}

OTH : Oesophagectomie transhiatale, GT : Gastrectomie totale, OGPS : Oesogastrectomie polaire supérieure, LS : Lewis Santy 